\title{
Human protein Staufen-2 promotes HIV-1 proliferation by positively regulating RNA export activity of viral protein Rev
}

\author{
Atoshi Banerjee ${ }^{1}$, Ronald Benjamin ${ }^{1}$, Kannan Balakrishnan ${ }^{1}$, Payel Ghosh² and Sharmistha Banerjee ${ }^{\text {** }}$
}

\begin{abstract}
Background: The export of intron containing viral RNAs from the nucleus to the cytoplasm is an essential step in the life cycle of Human Immunodeficiency Virus-1 (HIV-1). As the eukaryotic system does not permit the transport of intron containing RNA out of the nucleus, HIV-1 makes a regulatory protein, Rev, that mediates the transportation of unspliced and partially spliced viral mRNA from the nucleus to the cytoplasm, thereby playing a decisive role in the generation of new infectious virus particles. Therefore, the host factors modulating the RNA export activity of Rev can be major determinants of virus production in an infected cell.

Results: In this study, human Staufen-2 (hStau-2) was identified as a host factor interacting with HIV-1 Rev through affinity chromatography followed by MALDI analyses. Our experiments involving transient expressions, siRNA mediated knockdowns and infection assays conclusively established that hStau- 2 is a positive regulator of HIV-1 pathogenesis. We demonstrated that Rev-hStau-2 interactions positively regulated the RNA export activity of Rev and promoted progeny virus synthesis. The Rev-hStau-2 interaction was independent of RNA despite both being RNA binding proteins. hStau-2 mutant, with mutations at Q314R-A318F-K319E, deficient of binding Rev, failed to promote hStau-2 dependent Rev activity and viral production, validating the essentiality of this protein-protein interaction. The expression of this positive regulator was elevated upon HIV-1 infection in both human T-lymphocyte and astrocyte cell lines.
\end{abstract}

Conclusions: With this study, we establish that human Staufen-2, a host factor which is up-regulated upon HIV-1 infection, interacts with HIV-1 Rev, thereby promoting its RNA export activity and progeny virus formation. Altogether, our study provides new insights into the emerging role of the Staufen family of mRNA transporters in host-pathogen interaction and supports the notion that obliterating interactions between viral and host proteins that positively regulate HIV-1 proliferation can significantly contribute to anti-retroviral treatments.

Keywords: Staufen-2, Rev, RRE transport, HIV-1

\section{Background}

Human Immunodeficiency Virus-1 (HIV-1) is a pathogenic retrovirus that cripples the human immune system making it susceptible to several opportunistic infections and malignancies, leading to Acquired Immunodeficiency Syndrome (AIDS) [1-4]. Like all other successful intracellular pathogens, HIV also establishes dynamic biological interactions with the host cell machinery for persistence and pathogenesis. HIV-1, during the course of infection, makes completely spliced, partially spliced and unspliced mRNAs for

\footnotetext{
*Correspondence: sbs|@uohyd.ernet.in

1 Department of Biochemistry, School of Life Sciences, University of Hyderabad, Gachibowli, Hyderabad, Andhra Pradesh 500046, India Full list of author information is available at the end of the article
}

the synthesis of viral proteins to generate new virion particles [5]. While completely spliced viral mRNA utilizes the constitutive mRNA nuclear export pathway of the eukaryotic cells [6], the partially spliced and the unspliced viral mRNAs are retained in the nuclei of the infected host cells for further splicing or degradation. HIV overcomes this problem by synthesizing a protein called Rev (Regulator of virion expression) from completely spliced viral mRNA [7]. Rev is a $18 \mathrm{kDa}$ nucleo-cytoplasmic transport protein that tethers to Rev responsive element (RRE) present within the partially spliced and the unspliced viral mRNA and facilitate their export to the cytoplasm for the translation and progeny virus synthesis [8]. Rev, therefore, is an essential protein of the virus, the inactivation or 
deficiency of which results in failure to produce new virion particles [5].

Under physiological conditions, Rev shuttles between the cytoplasmic and the nuclear compartments where it interacts with several host factors that modulate its activity, consequently affecting HIV proliferation. Host nuclear proteins such as Matrin-3, RNA helicases, PSF, Rab/hRIP and CRM-1/exportin-1 that interact with Rev mediate its export from the nucleus to the cytoplasm [9-16]. Host proteins, such as, Ataxia-telangiectasia-mutated (ATM) protein [17], eukaryotic Initiation Factor-5a [18], Sam68 [19] and DEAD box RNA helicases [20], function as positive effectors of Rev activity, while factors such as, antiapoptotic mitochondrial protein, HS1-associated protein X-1 (Hax-1), negatively regulate the function of HIV-1 Rev by perturbing its sub-cellular distribution [21]. Some host proteins, which prevent effective binding of Rev to RRE, such as Rev Response Element Binding Protein of $49 \mathrm{kDa}$ (RREBP49) [22] or Alternate splicing factor (SF2/ASF) [23] interferes with the export activity of Rev, thereby reducing the virion particle formation. Apart from host factors, viral proteins also play a role in the sub-cellular localization of Rev which can eventually affect its activity [24]. An exhaustive list of the possible host factors interacting with HIV-1 has been reported by Jager et. al [25].

Acting as a post-transcriptional regulator of HIV-1 gene expression, identifying the cellular factors modulating Rev activity is highly pertinent to understand the pathogenicity of HIV-1. We hypothesized that cell specific host factors modulating the activity of Rev can serve as determinants in viral propagation and pathogenesis. Therefore, we attempted to capture Rev-interacting human cellular factors by making a Rev-affinity column, where purified recombinant histidine tagged HIV-1 Rev was used as bait. We characterized the interaction between Rev and a double-stranded RNA-binding host protein Staufen-2, identified from SUP-T1 (Human T cell lymphoblastoma) cell lysates. Human Staufen homolog 2 (hStau-2) is a double-stranded-RNA binding protein, with multiple splice variants [26] that possibly, like other mammalian Staufen homologs, forms ribonucleoprotein complexes (RNPs) [27]. hStau-2 has been shown to participate in targeted packaging and localization of mRNA in polarized cells and is a part of stress granules in neurons. $\mathrm{hStau}-2$ has also been observed in other cell types including cell lines like HeLa and $293 \mathrm{~F}[28,29]$.

In this study, the intracellular interactions between Rev and hStau- 2 were validated by transient expression of both the proteins in cell lines followed by coimmunoprecipitations. Ectopic and transient expressions, sub-cellular localizations, siRNA knockdowns and infection assays using wild type and mutant hStau-2 confirmed that the interaction of hStau-2 with Rev positively regulated the RRE containing RNA export activity of Rev and promoted progeny virus synthesis. Furthermore, HIV-1 infection elevated the expression of hStau-2 in both human T-lymphocyte and astrocyte cell lines. With these observations, we conclude that hStau-2, better known for its role in mRNA transportation and stress granule formation in neural cells [30], assumes a novel regulatory role during HIV-1 infection contributing to our existing knowledge of HIV-host interactions.

\section{Results}

Human Staufen-2 (hStau-2) interacted with HIV-1 Rev protein in RNA independent manner

Purified recombinant polyhistidine tagged HIV-1 Rev (Additional file 1: Figure S1A), immobilized on Talon resin, was used to pull-down Rev interacting host factors from SUP-T1 (Human T cell lymphoblastoma) cell lysates (Additional file 1: Figure S1B). An endogenously expressed double stranded RNA binding host protein, human Staufen-2 (hStau-2), was identified after MALDI analyses as one of the proteins in pull-down samples showing significant MASCOT score (Additional file 1: Figure S1C and Additional file 2: Figure S2). The interaction was further confirmed by co-immunoprecipitation of endogenously expressed hStau-2 from HEK293T cell lysates with transiently expressed GFP-tagged Rev (RevGFP). Rev-GFP was immunoprecipitated with mouse antiRev antibody and hStau-2 was detected by Western blot in the immunoprecipitated samples using goat anti-hStau-2 antibody (Figure 1A). HEK293T cells transfected with empty GFP vector was used as a control and did not show any hStau-2 signal (Figure 1A). The same was confirmed by immunoprecipitation with anti-GFP antibody followed by Western Blot with hStau-2 antibody (Figure 1B). These data clearly demonstrated that transiently expressed Rev could interact with the endogenous hStau-2 intracellularly in HEK293T cells.

hStau- 2 and Rev, both are RNA binding proteins. We, therefore, questioned if the cellular RNA mediated the Rev-hStau-2 interactions. For this, Rev-GFP and hStau-2 were transiently co-expressed in HEK293T cells and the cell lysates were divided into 2 aliquots, one of which was treated with RNase A. Total RNA from the treated and the untreated samples were isolated and fractionated on $2 \%$ agarose gel to check for the efficiency of RNase A treatment (Figure $1 \mathrm{C} 1^{\text {st }}$ panel). The treated and the untreated lysates were then used for immunoprecipitation with mouse anti-Rev antibody followed by Western blot with goat anti-hStau-2 antibody. It was observed that the degradation of ssRNA did not affect the interactions between Rev and hStau-2 (Figure 1C $2^{\text {nd }}$ and $3^{\text {rd }}$ panels). Since hStau-2 is a dsRNA binding protein and Rev is also reported to bind to the structured RRE element, we checked if the interaction is mediated by any dsRNA. For this, cell lysates were treated with RNase III which 

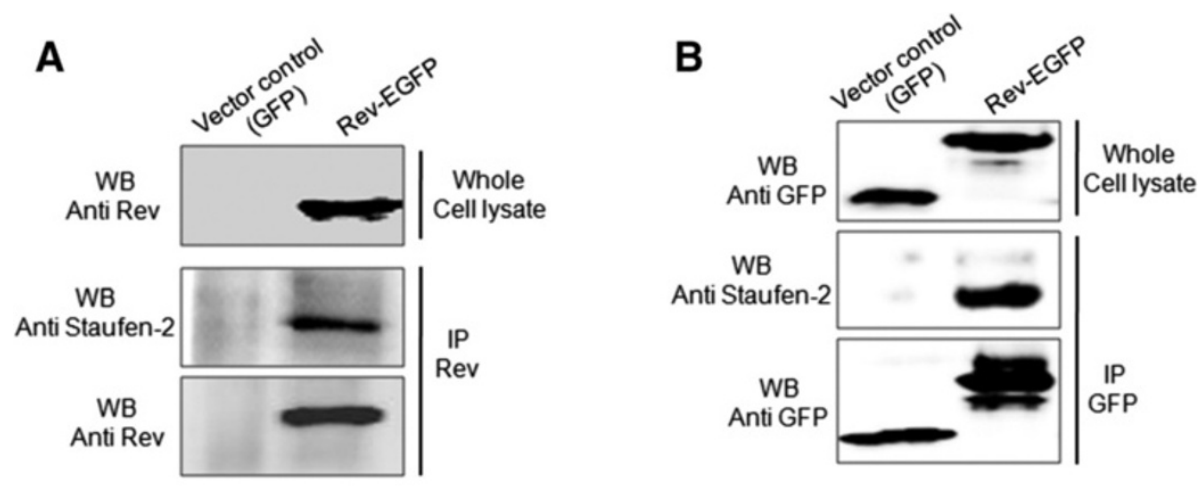

C

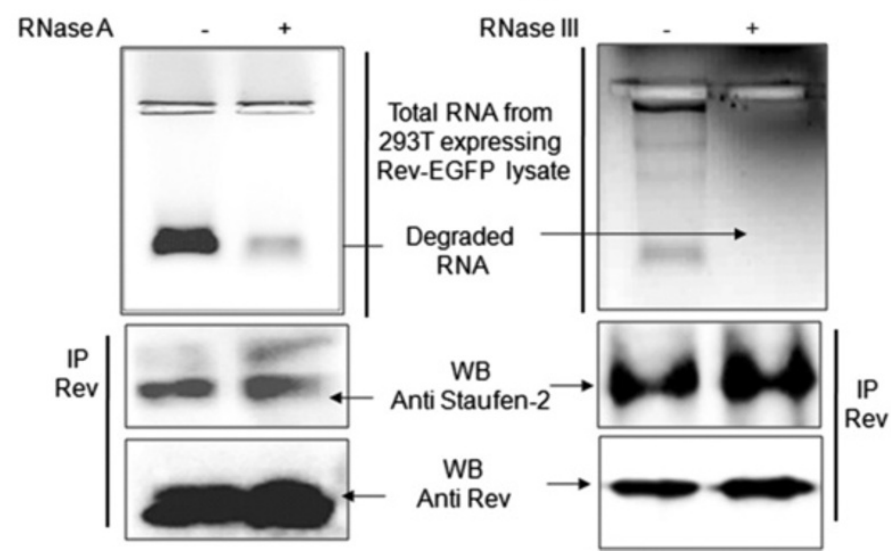

Figure 1 Rev interacts with hStau-2. A) Co-immunoprecipitation of endogenous hStau-2 with Rev-GFP using anti-Rev antibody and Western Blot with anti-hStau-2 antibody: HEK293T cells were transfected with Rev-EGFP vector or GFP vector control, cell lysates were prepared after 48 hours followed by IP and WB. Whole cell lysates were checked for Rev expression by anti-Rev antibody. B) Co-immunoprecipitation of endogenous hStau-2 with Rev-GFP using anti-GFP antibody and Western Blot with anti-hStau-2 antibody: HEK293T cells were transfected with Rev-EGFP vector or GFP vector control, cell lysates were prepared after 48 hours followed by IP and WB. Whole cell lysates were checked for GFP/Rev-GFP expression by anti-GFP antibody. C) RNase A treatment and pulldowns: Cell lysates were treated with RNase A. RNA degradation was assessed by RNA isolation from both RNase A untreated and treated cell lysates and run on 2\% Agarose gel ( $1^{\text {st }}$ Panel). Treated and untreated samples were used for IP with anti-Rev antibody and WB by anti-Staufen-2 antibody ( $2^{\text {nd }}$ Panel) and anti-Rev antibody ( $3^{\text {rd }}$ Panel). D) RNase III treatment and pulldowns: Cell lysates were treated with RNase III. RNA degradation was assessed by RNA isolation from both RNase III untreated and treated cell lysates and run on $2 \%$ Agarose gel ( $1^{\text {st }}$ Panel). Treated and untreated samples were used for IP with anti-Rev antibody and WB by anti-Staufen- 2 antibody $\left(2^{\text {nd }}\right.$ Panel) and anti-Rev antibody ( $3^{\text {rd }}$ Panel).

degrades double strand RNA (Figure 1D $1^{\text {st }}$ panel). Immunoprecipitations were performed as mentioned above and hStau-2 was detected in the immunoprecipitated samples (Figure 1D $2^{\text {nd }}$ and $3^{\text {rd }}$ panels). It was clear from this experiment that Rev and hStau-2 interaction is not mediated by dsRNA either. With this, we concluded that HIV-1 Rev interaction with hStau-2 is not RNA dependent.

\section{Rev influenced the sub-cellular localization of hStau-2}

Rev mediates the transport of RRE-containing viral RNA across the nuclear membrane by shuttling between the nucleus and the cytoplasm of an infected cell [31]. hStau-2, though primarily localizes in the cytoplasm, has also been shown to shuttle between the nucleus and the cytoplasm [26,32]. With both the proteins exhibiting nucleocytoplasmic shuttling properties, we addressed the question if Rev and hStau-2 co-localize in these sub-cellular compartments. The cellular localization of hStau- 2 alone and along with transiently expressed Rev-GFP was determined in HEK293T cells by confocal microscopy (Figure 2). When hStau-2 was overexpressed alone in HEK293T cells, it remained localized to the cytoplasm of the cells (Figure 2 A3-A4). However, when hStau-2 was co-expressed with Rev-GFP, a fraction of hStau- 2 was also observed to be localized in the nucleoli along with Rev (Figure 2 B3-B5). The localization of the two proteins was graphically represented by using twin slicer tool of Huygens Essential software as discussed in the methodology section [24]. The nuclear margins are indicated by blue (DAPI staining), hStau-2 is represented by Red (Alexa Fluor 568) and Rev is shown by green (GFP). The plots clearly show that hStau- 2 when expressed alone resides primarily in the cytoplasm while in 


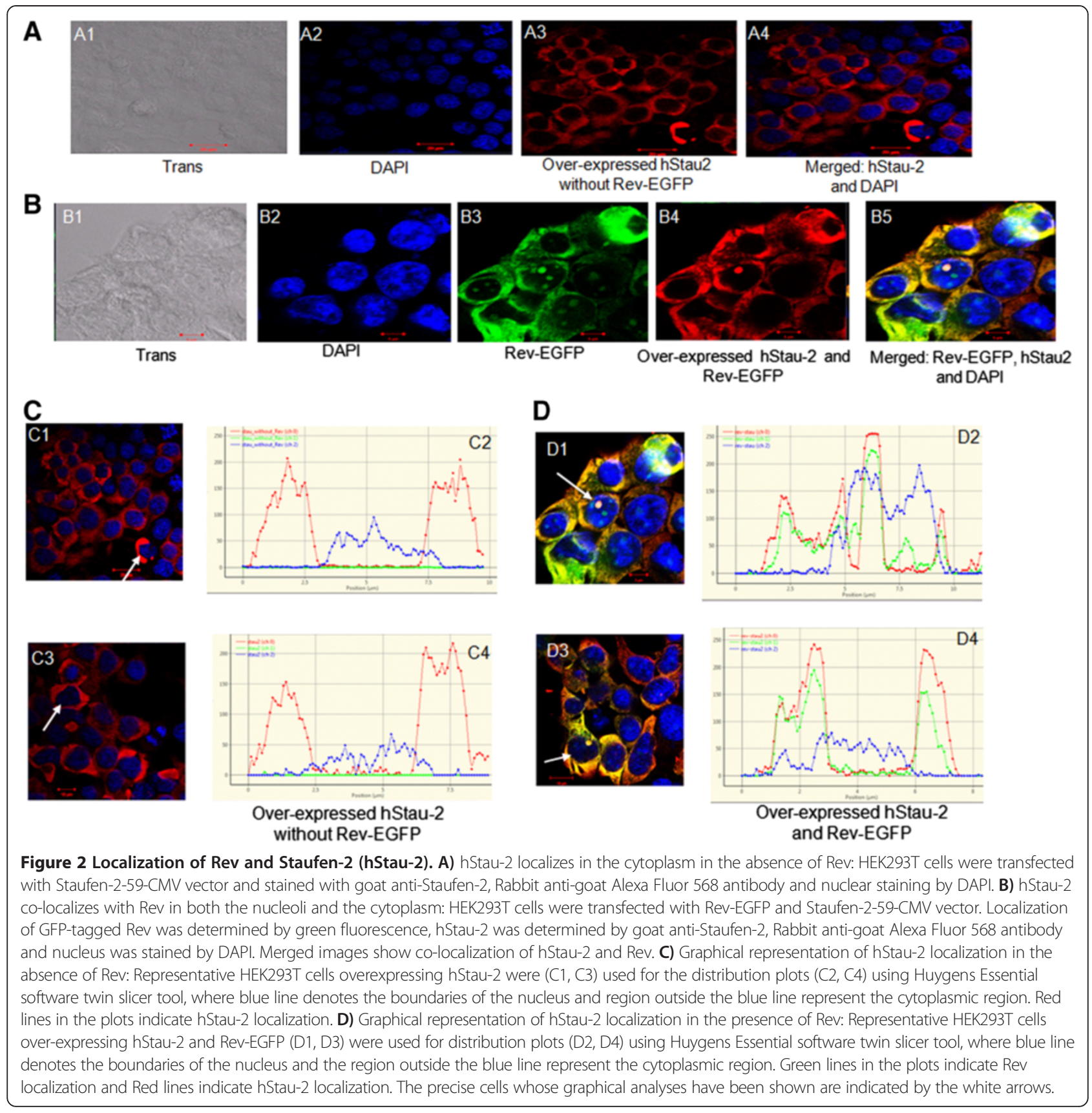

the presence of Rev it is found in both the nucleoli and the cytoplasm (Figure 2C-D). The merged Rev-GFP and hStau2 images (Figure 2 B5 and Figure 2D plots D2 and D4) show that the two proteins co-localize when expressed together. With this it was evident that the localization of $\mathrm{hStau}-2$, which was primarily cytoplasmic, was influenced in the presence of Rev.

HIV-1 infection up-regulated hStau-2 expression in human T-lymphocyte and astrocyte cell lines

Human Staufen-1 has been implicated in encapsidation and assembly of HIV-1 [33-35] whereas both the mam- malian homologs Staufen-1 and Staufen-2 are recruited in the stress granules of neural cells [30,36]. Apart from the neural cells, Staufen homologs have also been shown in other cell types $[28,29]$. Before any further characterization of Rev-hStau-2 interactions, we checked the fate of hStau-2 expression upon HIV-1 infection. Two HIV-1 permissive human cell-lines, SUP-T1 (Human $\mathrm{T}$ cell lymphoblastoma) and $1321 \mathrm{~N} 1$ (human astrocytoma) were used to verify the differential expression of hStau-2 upon HIV-1 (NL4-3) infection by quantitative Real time PCR. hStau-2 expression increased upon NL4-3 virus infection by $37 \pm 18 \%$ in SUP-T1 
(Figure 3A) while $60 \pm 15 \%$ in astrocyte $1321 \mathrm{~N} 1$ (Figure 3B) when compared to the mock infection. The transcript levels of $\beta$-actin were used for normalization of the data.

\section{hStau-2 increased HIV-1 production}

With the observation that hStau-2 expression is promoted during HIV-1 infection in human astrocyte and T-lymphocyte cell lines, we studied the influence of hStau-2 overexpression on HIV-1 viral titers. hStau-2 was transiently expressed in HEK293T cells 24 hours prior to transfection with pro-viral DNA pNL4-3. HEK293T cells were transfected with increasing concentration of hStau-2-59 construct and protein expression was checked by Western blot using anti-hStau-2 antibody (Figure $4 \mathrm{~A}$, inset). The viral titers were measured by p24 ELISA after 48 hours of pNL4-3 transfection. It was observed that the overexpression of hStau-2 significantly increased the viral titers in a dose dependent manner (Figure 4A). As compared to the control experiment, p24 equivalent of viral titers increased by $56.6 \pm$ $10.28 \%$ and $98 \pm 3.85 \%$ when hStau- 2 was overexpressed using $0.5 \mu \mathrm{g}$ and $1 \mu \mathrm{g}$ of hStau-2-59 construct respectively (Figure 4A). To check if the over-expression of hStau-2 is influencing the viral transcription and increasing the viral output, the total levels of full length viral transcripts were compared between hStau- 2 overexpressed and control experiments. No significant changes at the level of full length transcripts were observed with respect to the control in our experiments (Figure 4B). Hence, the impact of overexpression of hStau-2 on viral transcription was ruled out.

The effect of hStau-2 on viral titers was further confirmed by siRNA mediated knockdown of hStau- 2 . hStau-2 specific siRNA and scrambled siRNA were used for the study. hStau-2 specific siRNA effectively knocked down hStau-2 transcript levels as quantified through semi-quantitative RT-PCR (Figure 4C, inset). A dose dependent reduction in hStau-2 transcript levels was observed when 20 pmoles and 50 pmoles of hStau-2 specific siRNA were used as compared to scrambled siRNA (Figure 4C, inset). To check the consequences of hStau-2 knockdown on HIV progeny synthesis, HEK293T cells were transfected with pro-viral DNA pNL4-3 after 24 hours of silencing. Cells were harvested after 48 hours of pNL4-3 transfection and HIV-1 p24 levels from the culture supernatants were measured by p24 ELISA (Figure $4 \mathrm{C}$ ). The knockdown of hStau-2 resulted in a significant decrease in HIV-1 titers showing $30.75 \pm 8.9 \%$ and $57.32 \pm 11.82 \%$ reduction in p24 equivalent of viral titers in a dose dependent manner (Figure $4 \mathrm{C}$ ). Together, these data confirmed that hStau-2 behaved as a positive regulator of HIV-1 production.

\section{hStau-2 increased RRE containing viral mRNA export activity of Rev}

With the experiments confirming the promotion of HIV-1 production by hStau- 2 in addition to its direct interaction with Rev, we studied whether hStau-2 can enhance the RNA export activity of Rev. hStau-2 was overexpressed in HEK293T cells followed by transfection with pro-viral DNA pNL4-3. After 24 hours of transfection, the cells were harvested and cytoplasmic fractions were separated. Total RNA from the cytoplasmic fractions were extracted. The purity of cytoplasmic fraction was confirmed by the absence of pre-GAPDH mRNA in RT-PCR (Additional file 3: Figure S3). The levels of RRE containing viral RNA in the cytoplasm were then quantified by real time PCR using RRE specific primers. To quantify the RRE levels, a standard plot was generated based on the copy numbers of the amplified RRE region from the known concentration of RRE-GEM construct used as a template (Additional file 4: Figure S4A-B). Compared to the control experiments, hStau-2 overexpression promoted the export activity of Rev in a dose dependent manner as evident by 3 and 4 fold increase in the levels of RRE containing viral RNA in the
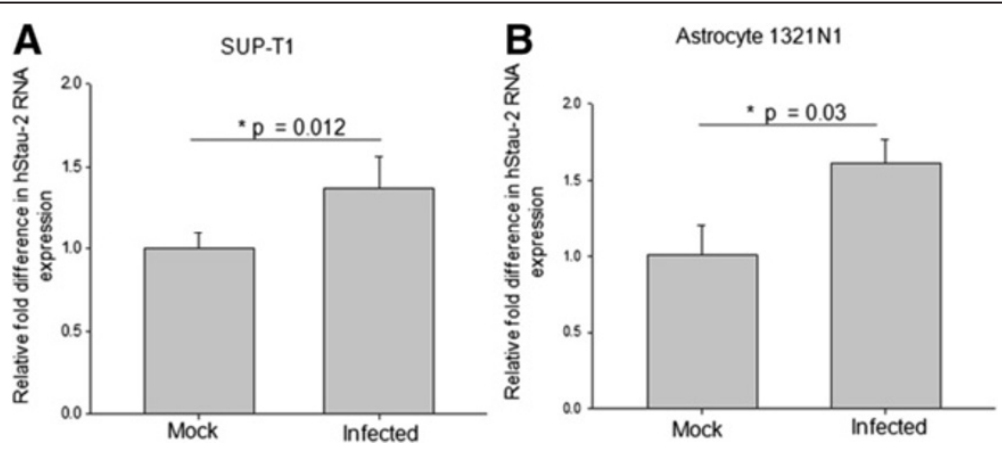

Figure 3 hStau-2 expression in SUP-T1 and 1321 N1 cell lines upon HIV-1 infection. Cells were infected with 50 ng/ml of p24 equivalent of NL4-3 virus. RNA was isolated from both mock and infected cells and qRT-PCR was carried out. A) hStau-2 expression in SUP-T1 cells B) hStau-2 expression in $1321 \mathrm{~N} 1$ cells. The result are from 4 independent experiments and error bars represent $\pm \mathrm{SD}$. ${ }^{*} \mathrm{p}$ value $\leq 0.05$ was taken as significant. The data were normalized to the transcript levels of $\beta$-actin. 


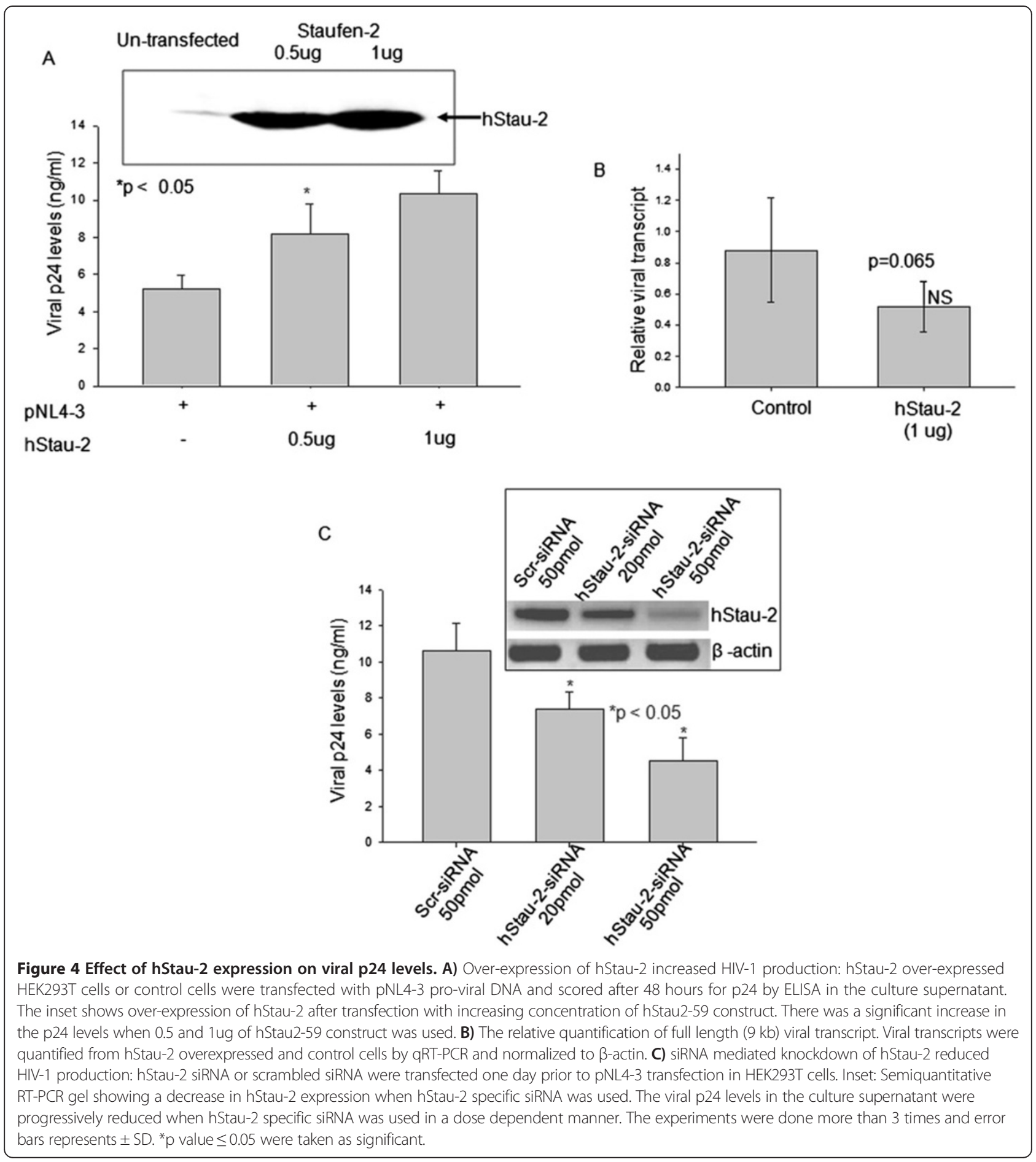

cytoplasmic fraction of HEK293T cells (Figure 5A). The CT values showed a significant shift towards the left with increasing amounts of hStau-2 expressing vector, indicating high copy number of RRE-RNA in the respective cytoplasmic fractions (Figure 5B).

To reconfirm that RRE-RNA export activity of Rev was indeed promoted by hStau- 2 , endogenous hStau- 2 was knocked down using siRNA and the above experiment was repeated. HEK293T cells treated with either scrambled siRNA or hStau-2 specific siRNA (in two increasing amounts) were transfected with equal amount of pNL4-3 pro-viral DNA. 24 hours post pNL4-3 transfection, the RRE-containing viral RNA was quantified from the cytoplasmic fractions of these cells by qRT- 
A
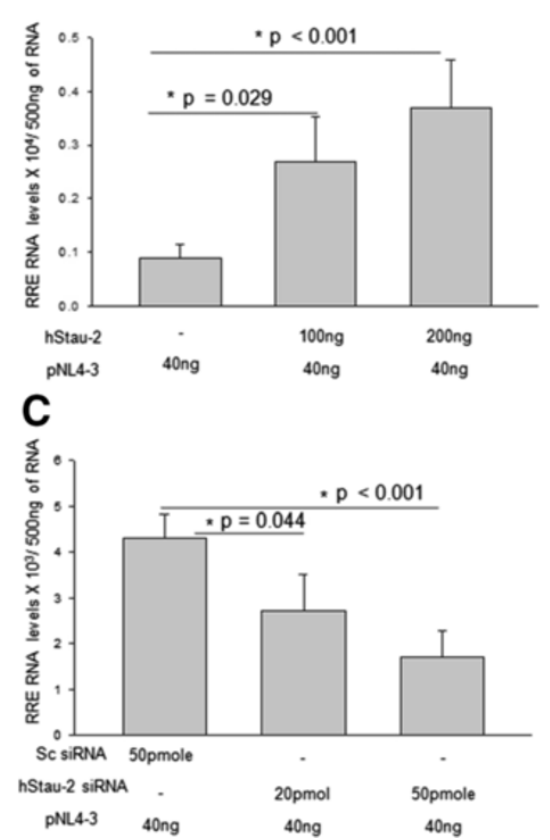

B

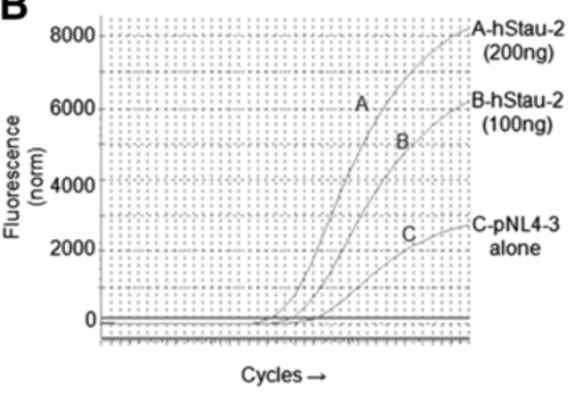

D

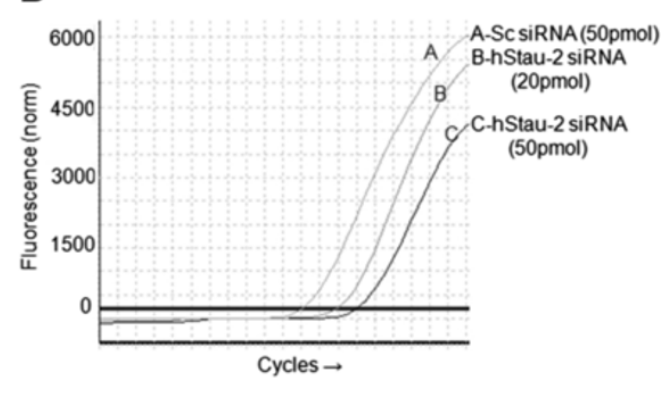

Figure 5 Effect of hStau-2 over-expression and knockdown on Rev export activity. A) Over-expression of hStau-2 increased RRE-RNA levels in the cytoplasmic fraction of HEK293T cells: hStau-2 over-expressed HEK293T cells or control cells were transfected with pNL4-3 pro-viral DNA. After 24 hrs of pNL4-3 transfection, total RNA was isolated from the cytoplasmic fractions and cDNA was prepared from 500 ng of RNA. Viral RRE was measured in the cytoplasmic fraction by absolute qRT-PCR. B) A leftward shift in CT curves for RRE-containing RNA upon hStau-2 overexpression. C) hStau-2 knockdown reduced RRE-RNA levels in the cytoplasmic fraction of HEK293T cells: hStau-2 siRNA or scrambled siRNA were transfected into HEK293T cells followed by pNL4-3 pro-viral DNA transfection. After 24 hrs of pNL4-3 transfection, total RNA was isolated from the cytoplasmic fractions and CDNA was prepared from $500 \mathrm{ng}$ of RNA. Viral RRE was measured in the cytoplasmic fraction by absolute qRT-PCR. D) A rightward shift in CT curves for RRE-containing RNA upon hStau-2 knockdown. The experiments were done more than 3 times and the error bar represents \pm SD. ${ }^{*} \mathrm{p}$ value $\leq 0.05$ was taken as significant.

PCR and the CT values were compared (Figure 5C-D). As clear from the plot in Figure 5C, RRE containing viral RNA in the cytoplasm of the cells were 1.5 and 2.5 fold less in conditions where hStau-2 was knocked down using increasing doses of siRNA (Figure 5C). As expected, a significant right shift in CT curves for RREcontaining RNA in hStau-2 knocked down cells were observed when compared to the scrambled siRNA treated cells (Figure 5D). Collectively, these experiments established that the host protein hStau-2, which was upregulated during HIV-1 infection, promoted RRE containing RNA export activity of Rev and increased HIV-1 titers.

\section{hStau-2 mutant defective in Rev interaction failed to} promote RNA export activity of Rev and viral production The experiments so far indicated that the presence of hStau-2 positively regulated Rev RNA export activity and the viral production. To confirm that the physical interaction between hStau-2 and Rev is instrumental in promoting Rev activity, a hStau-2 mutant (referred as hStau-2Mut) defective in Rev binding was generated. For this, HIV-1 Rev was docked onto available Staufen-2 homolog to predict the critical residues of hStau-2 that possibly interacted with Rev. The best ranked complexes from three different docking studies (Additional file 5: Figure S5 A-B) suggested that hStau-2 residues spanning 314-320 are playing an important role in the interaction with Rev (Figure 6A). Based on these predictions, hStau2Mut with mutations Q314R, A318F and K319E was generated. This mutant was used in the co-immunoprecipitation assays to check for its interaction with Rev. Rev-GFP was co-overexpressed with either the wt hStau2 or hStau-2Mut. The cell lysates were confirmed for the overexpression of Rev, wt hStau-2 and hStau2 Mut by Western blots (Figure $6 \mathrm{~B}, 1^{\text {st }}$ and $2^{\text {nd }}$ panel). Immunoprecipitations were performed using mouse anti-Rev antibody. hStau-2 was detected by Western blot in the immunoprecipitated samples using goat anti-hStau-2 antibody (Figure $6 \mathrm{~B}, 3^{\text {rd }}$ panel). It was observed that mutations, Q314R, A318F and K319E, did not obliterate the Rev-hStau-2 interactions completely, though made it defective for Rev binding (Figure 6B).

In order to check if hStau-2Mut defective for Rev binding had different sub-cellular distribution than the wt hStau-2, we observed its localization in the presence or in the absence of Rev by confocal microscopy 

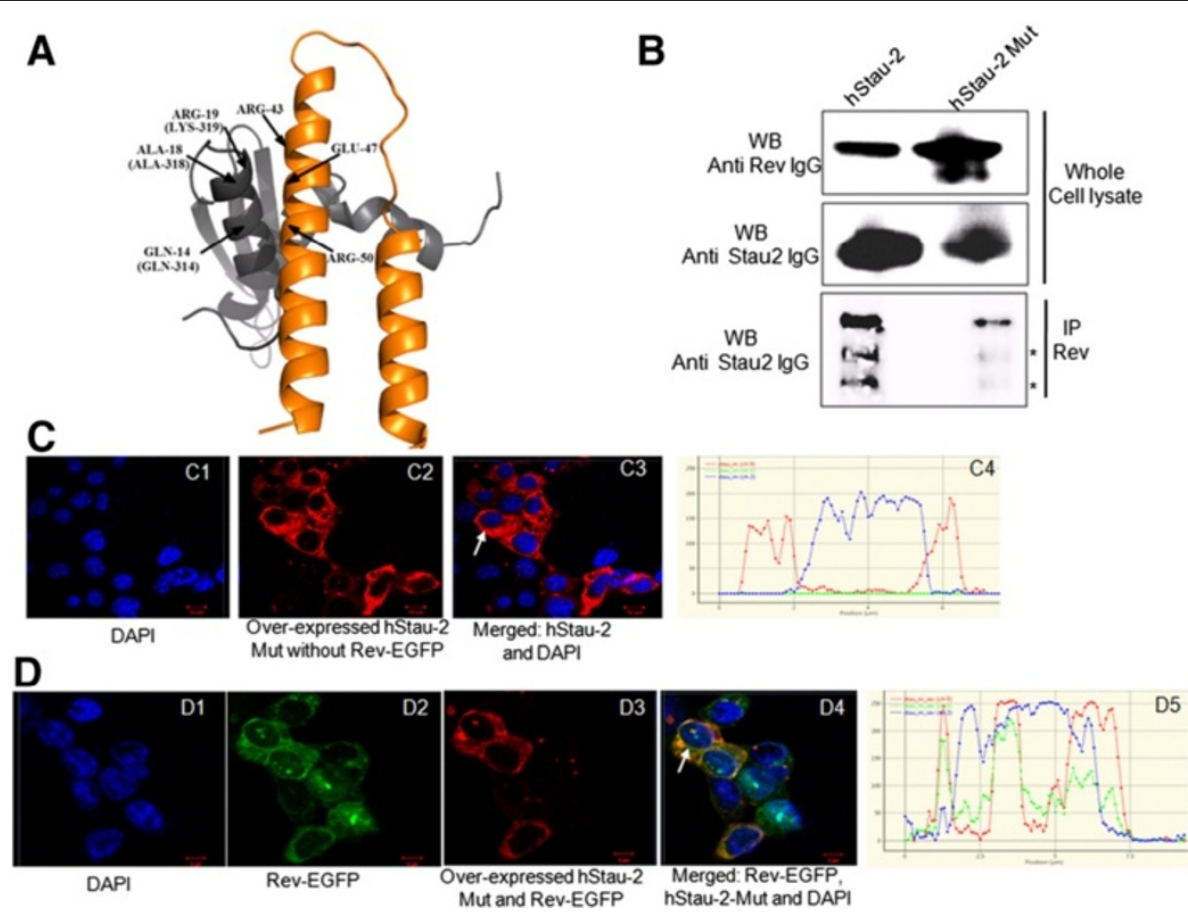

Figure 6 Rev-hStau-2 interactions are essential for hStau-2 dependent promotion of Rev activity. A) The docked complex of Staufen homolog from mouse ( $1 \mathrm{UHZ}$, grey ribbon) and Rev protein ( $2 X 7 \mathrm{~L}$, orange ribbon) showing interacting residues: The corresponding residues of hStau-2 protein are indicated in brackets. B) Co-immunoprecipitation of hStau-2 or hStau-2Mut with Rev using anti-Rev antibody: HEK293T cells were transfected with Rev-EGFP vector and co-transfected with hStau2-59 or hStau-2Mut-CMV vector, cell lysates were prepared after 48 hours followed by IP and WB. Whole cell lysates were checked for Rev expression by anti-Rev antibody and for hStau-2 expression by anti-hStau-2 antibody. ${ }^{*}$ indicates non-specific bands or degraded protein. C) Localization of hStau-2Mut. hStau-2Mut localizes in the cytoplasm: HEK293T cells were transfected with hStau-2Mut-CMV vector and stained with goat anti-Staufen-2, Rabbit anti-goat Alexa Fluor 568 antibody and nucleus was stained by DAPI. Distribution plot is shown at the end of the panel. D) Co-localization of Rev and hStau-2Mut: HEK293T cells were transfected with Rev-EGFP and hStau-2Mut-CMV vector. Localization of GFP-tagged Rev was determined by green fluorescence, hStau-2Mut was determined by goat anti-Staufen-2, Rabbit anti-goat Alexa Fluor 568 antibody and nucleus was stained by DAPI. Merged images show co-localization of hStau-2Mut and Rev. Distribution plot is shown at the end of the panel. The precise cells whose graphical analyses have been shown are indicated by the white arrows.

(Figure 6C-6D). Pattern of distribution of hStau-2Mut in the sub-cellular regions of the nucleus and the cytoplasm was similar to that of the wild type hStau-2. Similar to the wild type hStau-2, hStau-2Mut localized primarily to the cytoplasm in the absence of Rev while a fraction of it localized to the nucleoli in the presence of Rev (Figure 6C-6D). These studies clearly indicated that though hStau-2Mut was defective in binding to Rev, other properties like the sub-cellular localization was not affected.

We then scored the influence of hStau-2Mut on the RNA export activity of Rev. The levels of RREcontaining viral RNA in the cytoplasm of HEK293T cells transfected with pNL4-3, overexpressing either the wildtype (hStau-2) or the mutant (hStau-2Mut) hStau-2 were studied using qRT-PCR (Figure 7A). It was evident that the cytoplasm of HEK293T cells over-expressing hStau-2 had higher levels of RRE-containing viral RNA compared to the control experiment (pNL4-3) denoting Rev-supporting function of hStau-2, while the levels of
RRE-containing viral RNA in the cytoplasm of HEK293T cells that over-expressed hStau-2Mut remained similar to that of the control experiment (Figure 7A). The CT curve for RRE-containing RNA for hStau-2 showed a significant left shift with reference to the CT curve for the control experiment (Additional file 6: Figure S6 curves A and C), while no such shift was observed for hStau-2Mut (Additional file 6: Figure S6 curve B). From these results, it was inferred that hStau-2 dependent promotion of Rev activity was not exhibited by hStau-2Mut that was defective in interacting with Rev. Further, when p24 equivalent of HIV-1 titers was measured in the culture supernatants from similar experiments, it was observed that hStau- 2 over-expressing cells produced more viruses than the control experiment, while cells expressing hStau-2Mut produced less virus particles, even lesser than that produced in the control experiment (Figure 7B). These experiments confirmed that the protein-protein interaction between Rev and hStau-2 was important for the modulation of Rev-export activity and viral titers. 


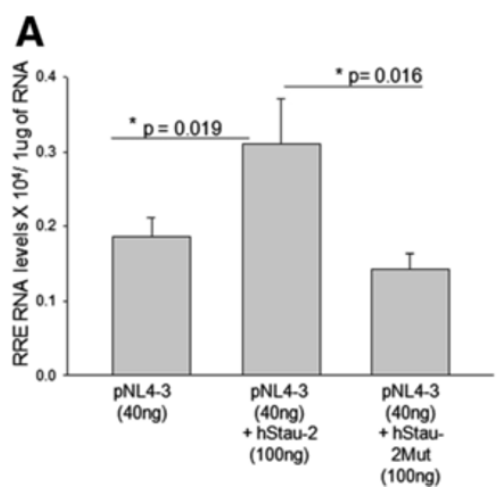

\section{B}

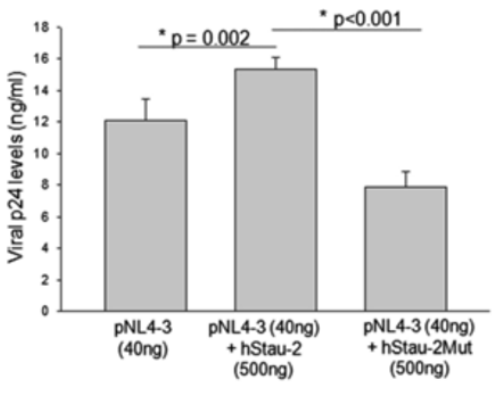

Figure 7 Rev-hStau-2 interactions are essential for hStau-2 dependent promotion of Rev activity. Viral RRE-RNA levels were measured in the cytoplasmic fraction by absolute qRT-PCR for either hStau-2 or hStau-2Mut over-expressing cells. A) hStau-2Mut did not promote RRE-RNA export activity of Rev: Viral RRE-RNA levels were measured in the cytoplasmic fraction by absolute qRT-PCR for hStau-2, hStau-2Mut over-expressing cells and the control cells. Concentrations of the plasmid hStau-2 or hStau-2-Mut was 0.1ug and pNL4-3 was 40 ng. B) Viral p24 levels were scored by p24 ELISA. Concentration of the plasmid hStau-2 or hStau-2-Mut was 0.5ug and pNL4-3 was 40 ng. hStau-2Mut did not increase HIV-1 production.

\section{Discussion}

This study describes a new host factor, Staufen-2, that assists in HIV-1 pathogenesis by supporting the activity of a critical viral regulatory protein, Rev, thereby increasing the viral production in an infected cell. The observations were supported by over-expression and siRNA mediated knockdown of hStau-2, where it was evident that hStau-2 increased the RRE-RNA export activity of Rev and the viral titers (Figures 4 and 5). Abrogating the direct interaction between Rev and hStau- 2 by generating hStau-2 mutant defective for Rev binding, abolished hStau-2 dependent promotion of Rev activity and hence final viral output. This positive regulator of HIV-1 was also observed to be upregulated during HIV-1 infection of human T-lymphocyte and astrocyte cell lines (Figure 3A-B).

Cellular protein, Staufen belongs to dsRNA binding protein family, first identified in Drosophila [37]. Mammals possess two Staufen homologs, Staufen 1 and Staufen 2, each with multiple isoforms and splice variants [38]. Both the Staufen homologs are reported to form RNA-protein complexes and participate in transport, stability and decay of mRNA in human neurons or other polarized cells thereby regulating the spatial and the temporal gene expressions [30,39]. Recently, it was deciphered that Staufen 1 , despite being a host protein, is selectively packaged in HIV-1 infectious particle through its interaction with HIV-1 Gag [34,35], while there were no previous reports of hStau- 2 being involved in any phase of the HIV-1 life cycle. It was intriguing to identify hStau- 2 in the pulldown samples of Rev as we noticed that Rev, a protein from the pathogen and hStau-2, a protein from the host, had overlapping biochemical and functional properties. Both the proteins bind RNA, shuttle between the nucleus and the cytoplasm and mediate mRNA transportation across the nuclear membranes. Earlier reports by independent studies showed that CRM1/Exportin-1 can interact separately with both Rev and hStau-2 and promote their RNA export activity [32,40]. With our studies showing that Rev and hStau-2 physically interacted with each other (Figure 1A and B) and that this interaction promoted the Rev activity (Figure 5), it can be speculated that together with CRM1 and hStau-2, Rev may form an elaborate export complex for the successful transportation or regulated release of large viral RNA across the nuclear membrane. It should be noted that the composition of such multiprotein-RNA complexes may vary across cell types and would be interesting to explore how the composition of such complexes impact the viral RNA transport by Rev.

A number of RNA binding host proteins like RNA helicase A (RHA), human Rev-interacting protein (hRIP) etc., interact with Rev and influence its activity [15,41,42]. These interactions are either direct or mediated by other proteins or RNA. In case of hStau-2, it could be established that the interaction with $\mathrm{Rev}$ is independent of RNA (Figure 1C-1D). Though this strongly suggested a direct protein-protein interaction, we cannot rule out the possibility of cellular or viral RNA existing between the two proteins under physiological conditions. Yet another interesting fact to further decipher would be to identify the respective RNA partners of hStau- 2 and Rev under varied physiological conditions in different HIV permissive host cells.

In agreement with the positive regulation of hStau-2 on Rev activity, over-expression of hStau-2 increased viral titers. The essentiality of the direct protein-protein interaction between Rev-hStau-2 was established through hStau-2Mut defective in Rev binding that did not support the hStau-2 dependent promotion of Rev activity and increase in the viral titers (Figure 7A-B). In fact, the cells overexpressing hStau-2Mut produced lesser virus than pNL4-3 alone transfected control cells (Figure 7B). These 
observations are suggestive of multiple roles of hStau- 2 in the life cycle of HIV-1 apart from increasing Rev mediated viral RNA export activity. These studies also demonstrated that disrupting the protein-protein interaction between Rev and hStau-2 can prevent hStau-2 from helping viral production. This information holds high significance as the Rev-Staufen2 docking studies (Figure 6A and Additional file 5: Figure S5) can be further exploited to design peptide based retroviral inhibitors where disrupting Rev-hStau-2 interactions would limit viral propagation.

Staufen containing RNA granules are a part of high molecular mass (HMM) complexes of cytoplasmic nucleoproteins. Low Molecular Mass (LMM) A3G when gets converted into HMM loses its antiretroviral property and cannot limit HIV-1 propagation in infected cells $[43,44]$. In our observations, hStau-2 expression was upregulated upon HIV-1 infection of CD4 + T lymphocyte cell line SUP-T1. It can be inferred that T-cells, when activated by HIV-1 infection expressed more hStau-2 that further helped making more Staufen containing HMM complexes that prevented endogenous LMM-A3G from inhibiting HIV propagation. This can be one more approach to explain the high viral production by the infected cells over-expressing hStau-2 apart from the proRev function of hStau-2. HIV-1 infection upregulated hStau-2 expressions in astrocytes as well (Figure 3B). It would be interesting to further study the impact of increased levels of hStau-2 on neuronal cells. Distorted hStau-2 levels in these cells upon HIV infection may alter mRNA transport and hence protein expressions that can explain conditions like HIV associated neuro-dementia.

This study has established the involvement of yet another member of the Staufen family of protein in HIV pathogenesis, where hStau-2 regulated HIV-1 titers through direct interaction with one of the key viral proteins. It is evident from our study that one possible mechanism by which hStau-2 promoted viral production is by aiding HIV-1 protein Rev in transporting viral RNA for the synthesis of late viral structural proteins. Further investigations to elucidate the mode of action of hStau-2 in context of HIV pathogenesis will add substantial information for designing new anti-retroviral strategies.

\section{Conclusion}

Conclusively, we have identified host protein hStau-2 as a positive effector of HIV-1 Rev that promoted its RNA export activity, thereby facilitating viral production, the expression of which was upregulated upon HIV-1 infection.

\section{Methods}

Cell lines, viral stock production and HIV-1 infection

The cell lines used in the study: HEK293T (human embryonic kidney cell line) obtained from Dr. Reddy's
Institute of Life Sciences, Hyderabad and Astrocyte 1321 N1 human astrocytoma (gift from Dr. Kondapi) were maintained in DMEM media (Invitrogen, USA). SUP-T1 (human $\mathrm{T}$ cell lymphoblastoma) obtained from Dr. S. Jameel, ICGEB Delhi was maintained in RPMI-1640 media (Invitrogen, USA). Additionally, the media were supplemented with $10 \%$ fetal bovine serum (Gibco, USA), $100 \mathrm{U}$ of penicillin/ml and $100 \mu \mathrm{g}$ of streptomycin/ml (Himedia Laboratories, India). All the cell lines were grown in $37^{\circ} \mathrm{C}$ with $5 \% \mathrm{CO}_{2}$.

Infectious HIV-1 particles were produced by transfection of proviral DNA pNL4-3 into HEK293T cells by calcium phosphate method [45]. Culture supernatants were collected at an interval of 24 hours post transfection, filtered through $0.45 \mu \mathrm{M}$ syringe filter (Millipore, USA), precipitated using Polyethylene glycol [45] and quantified by HIV-1 p24 ELISA kit (Advanced BioScience Laboratories Inc, USA) according to the manufacturer's protocol. The virus thus produced is HIV type 1 (HIV-1) referred as NL4-3. Cells were infected with $50 \mathrm{ng} / \mathrm{ml}$ of p24 equivalent of NL4-3 virus in the presence of $8 \mu \mathrm{g} /$ $\mathrm{ml}$ of polybrene (Sigma-Aldrich, USA). After 4 hours of infection, cells were washed with phosphate buffer saline (PBS) and kept for 48 hours in complete media. The protocol used and standards maintained are approved by Institutional Biosafety Committee, University of Hyderabad.

\section{Plasmids and constructs \\ His-Rev-setB}

Full length cDNA of HIV-1 Rev was amplified from CMVsrev (gift from Prof. B. K. Felber, Center for Cancer Research, USA) using primers Rev-His-FP and Rev-His-RP (Additional file 7: Table S1) and cloned into BglII and NcoI sites of pRSET-B E.coli T7 Expression Vector (Invitrogen, USA) to generate hexa-histidine tag at the N-terminal.

\section{Rev-EGFP-C3}

The Rev gene was amplified from His-Rev-setB construct using primers Rev-GFP-FP and Rev-GFP-RP (Additional file 7: Table S1) and cloned into BglII and Sall site of pEGFP-C3.

\section{hStau-2Mut-CMV}

hStau-2-59 (gift from Prof. Luc Desgroseillers, Université de Montréal, Canada) was used as a template to create Human Staufen 2 mutant. The mutant region containing Q314R, A318F and K319E mutations were generated by overlap PCR using Stau-2Mut FP and Stau-2Mut RP (Additional file 7: Table S1) and cloned into SalI and XbaI site of pCMV-Sport 1 (Invitrogen, USA).

\section{RRE-GEM}

Using pNL4-3 as a template, RRE sequence was cloned into EcoRI and HindIII sites of pGEM-3Zf + (Promega, 
USA) vector. The primer sequences used were RRE-FP and RRE-RP (Additional file 7: Table S1).

All the constructs were confirmed by sequencing (Eurofins, India).

Other plasmids used were hStau-2-59 (gift from Prof. Luc Desgroseillers, Université de Montréal, Canada) for expression of wild type hStau-2, pro-viral DNA construct pNL4.3 (gift from Prof. A. K. Kondapi, University of Hyderabad, India), pEGFP-C3 (Clonetech, USA).

\section{Expression and purification of the recombinant Histidine tagged Rev protein}

His-Rev-setB construct was transformed into BL-21 DE3 codon plus (RIL) cells (Agilent technologies, USA) for expressing recombinant Rev protein with $\mathrm{N}$ terminal $6 \mathrm{X}$ histidine tag by induction with $1 \mathrm{mM}$ of Isopropyl $\beta$-D-1thiogalactopyranoside (IPTG) (Fermentas, Germany) for 6 hours at $37^{\circ} \mathrm{C}$. Rev was purified under native conditions using Talon-affinity resin (Clontech, USA) according to the manufacturer's protocol and eluted with $150 \mathrm{mM}$ imidazole. After purification, Rev protein was dialyzed in modified Rev-buffer [46] with the composition of $50 \mathrm{mM}$ sodium phosphate buffer, $\mathrm{pH}$ 7.0, $150 \mathrm{mM} \mathrm{NaCI}, 10 \mathrm{mM}$ $\mathrm{K}_{2} \mathrm{SO}_{4}$, and $1 \mathrm{mM}$ DTT. The protein was concentrated using centricon with $3 \mathrm{kDa}$ cutoff (Millipore, USA). Protein concentration was measured by $1 \mathrm{X}$ Bradford Dye Reagent (Bio-Rad, USA) according to the manufacturer's instructions. The purified protein was checked on 15\% SDS PAGE (Additional file 1: Figure S1A) and confirmed by Western blot using 1:2000 dilution of mouse anti-Rev antibody (SantaCruz Biotechnology Inc., USA) and 1:2500 dilution of HRP conjugated goat anti-mouse IgG antibody (SantaCruz Biotechnology Inc., USA) followed by detection with Pierce ECL western blotting substrate (Thermo Scientific, USA) and visualized using VersaDoc gel imaging system (BioRad, USA).

\section{Affinity chromatography to identify Rev interacting host factors}

Affinity column was prepared using recombinant His-Rev protein according to the previously described protocol [47] with some modifications. In brief, $100 \mu \mathrm{g}$ of recombinant His-Rev was added to the $500 \mu \mathrm{l}$ of Talon resin and incubated for 4 hours at $4^{\circ} \mathrm{C}$. Wash with Rev buffer (50 mM sodium phosphate buffer, $\mathrm{pH}$ 7.0, $150 \mathrm{mM} \mathrm{NaCI}$, $10 \mathrm{mM} \mathrm{K}_{2} \mathrm{SO}_{4}$, and $1 \mathrm{mM} \mathrm{DTT}$ ) was given to remove the unbound Rev protein. SUP-T1 cells were lysed by lysis buffer (50 mM Tris- $\mathrm{HCl}$ pH 7.5, $150 \mathrm{mM} \mathrm{NaCl}, 2 \%$ Glycerol, $5 \mathrm{mM}$ DTT, 0.1\% NP-40, $1 \mathrm{mM}$ PMSF and protease inhibitor cocktail), centrifuged to remove debris and supernatant from which $1 \mathrm{mg}$ of total protein was added to the Rev bound Talon resin. As a control, SUP-T1 cell lysates were added to the column without bound recombinant His-Rev protein. After overnight incubation, columns were washed 5 times with wash buffer (Rev buffer $+50 \mathrm{mM}$ imidazole). The samples were eluted using elution buffer (Rev buffer $+150 \mathrm{mM}$ imidazole). $40 \mu \mathrm{l}$ of eluted samples from both test and control columns were subjected to SDS PAGE followed by silver staining [48]. Bands unique to Rev affinity column pull-down were excised (Additional file 1: Figure S1B) and identified using MALDI-TOF (Bruker Daltonics, Bremen, Germany) at proteomics facility of the University of Hyderabad, India. Protein bands were identified using database searches (PMF and MS/MS) using MASCOT program by Biotools software (Bruker Daltonics) (Additional file 1: Figure S1C and Additional file 2: Figure S2).

\section{Immunoprecipitations}

Transfected HEK293T cells were washed with PBS and lysed in IP-Buffer [20 mM Tris $\mathrm{HCl} \mathrm{pH} 8.0,137 \mathrm{mM}$ $\mathrm{NaCl}, 10 \%$ glycerol, 1\% Nonidet P-40 (NP-40)]. Cells were sonicated and centrifuged at $12000 \mathrm{rpm}$ at $4^{\circ} \mathrm{C}$ to remove cellular debris. For RNA digestion, cell lysates were treated either with RNase A or with RNase III as per the manufacturer's protocol. Agarose A/G beads (SantaCruz Biotechnology Inc., USA) were washed with IP buffer and incubated with cell lysates for 2 hours at $4^{\circ}$ $\mathrm{C}$ to remove proteins that non-specifically bind to the beads (pre-clearing step). After pre-clearing, the beads were centrifuged at $4000 \mathrm{rpm}$ and the supernatant was added to the new column containing A/G beads already conjugated with mouse anti-Rev antibody or anti-GFP antibody for 4 hours at $4^{\circ} \mathrm{C}$ on a rocker. After 4 hours of incubation, samples were washed 3 times with IP buffer. The samples were processed for Western blot.

\section{Western blot}

Samples were fractionated on 10\% SDS-PAGE and transferred to methanol treated PVDF membrane (GEAmersham, USA) in a Western blot transfer apparatus. After the membranes were blocked with 5\% non fat milk for 1 hour at RT, the primary antibody was added to the membrane and incubated overnight at $4^{\circ} \mathrm{C}$ on a rocker. The antibody dilution used for mouse anti-Rev antibody was 1:1000 (SantaCruz Biotechnology Inc., USA), for mouse anti-GFP antibody was 1:1000 (SantaCruz Biotechnology Inc., USA) and for goat anti-Stau-2 antibody was 1:500 (SantaCruz Biotechnology Inc., USA). After 3 washes with PBS-T (PBS + 0.05\% Tween 20), the membranes were incubated with the respective HRP conjugated antibodies namely, goat anti mouse IgG with 1:2000 dilution (SantaCruz Biotechnology Inc., USA) and donkey anti-goat IgG with 1:1000 dilution (SantaCruz Biotechnology Inc., USA) for respective detection of Rev, GFP and hStau-2 at RT for 1 hour. Following 3 washes with PBS-T, ECL substrate (Super Signal West 
Femto Chemiluminescent Substrate Pierce) was added to the membranes and was scanned using Versadoc system (Biorad). The Western blot images were quantified by Image-J software (NIH).

\section{Immuno-fluorescence microscopy}

For fluorescence studies, cells were grown on coverslips one day before transfection. Cells were transfected with respective plasmids using lipofectamine LTX plus reagent (Invitrogen, USA). After 48 hours of transfection, cells were washed twice with PBS and fixed in 3\% paraformaldehyde. Fixed cells were washed 3 times with PBS to remove excess of paraformaldehyde and permeabilized by adding ice-cold methanol for $5 \mathrm{~min}$ at $-20^{\circ} \mathrm{C}$. Cells were washed thoroughly and mounted with DAPI. Rev-GFP exhibited green fluorescence. hStau-2 was detected using polyclonal anti-hStau- 2 antibody and corresponding secondary fluorescent-tagged, Alexa Fluor 568 rabbit anti-goat IgG antibody (red). The nuclei of the cells were stained with DAPI (blue). Cells were seen under Leica confocal TPS2 microscope at 20X magnification and analyzed using Leica software. For localization in the cytoplasm or the nucleus, several fields were scanned and Huygens Essential software was used to plot graph representing fluorescence intensities of blue (for nucleus), green (for Rev) and red (for Staufen-2) channels across the cells using twin slicer tool [24].

\section{Quantitative Real time PCR}

RNA was isolated using Trizol reagent (Invitrogen, USA) according to the manufacturer's protocol. cDNA was prepared from RNA by using Superscript III (Invitrogen, USA) and oligo dT (Fermentas, Germany ) at $50^{\circ} \mathrm{C}$ for 1 hour followed by inactivation at $70^{\circ} \mathrm{C}$ for $15 \mathrm{~min}$. hStau2 and $\beta$-actin genes were amplified by SYBR Premix ExTaq (Takara Bio Inc, Japan) in Eppendorf Mastercycler Realplex $^{2}$ (Eppendorf, Germany). The fold difference was calculated using the formula $2^{-(\Delta \Delta C T)}$. The primers used were Stau-RT-FP, Stau-RT-RP, Actin-RT-FP and Actin-RT-RP mentioned in Additional file 7: Table S1.

\section{Transfection and silencing}

HEK293T cells were seeded one day before transfection at $70 \%$ confluency. Cells were washed with PBS and transfected with plasmids using Lipofectamine-LTX plus reagent (Invitrogen, USA). After 48 hours of transfection, cells were harvested.

To silence hstau-2 gene, a 21 bp duplex siRNA was synthesized for a selected region of hStau-2. As a control, scrambled siRNA was used. hStau-2 specific siRNA (20 and 50 picomoles) or scrambled siRNA (50 picomoles) were transfected using Lipofectamine RNAiMAX reagent (Invitrogen, USA). The synthesized siRNA were tested for knockdown efficiency in preliminary experiments by semi-quantitative RT-PCR using Stau-RT-FP and StauRT-RP primers for 25 cycles (Additional file 7: Table S1). $\beta$-actin was used as a control for the experiment. The reaction without reverse transcriptase was used as a negative control to confirm that RNA preparation is free of DNA contamination.

After 24 hours of siRNA treatment, cells were transfected with pNL4-3 plasmid and kept for additional 48 hours.

\section{Quantification of total viral transcripts}

For quantification of total viral RNA transcripts, equal amount of cDNA was used to amplify $9 \mathrm{~kb}$ product by qRT-PCR using primer set TAR-FP and $9 \mathrm{~kb}-\mathrm{RP}$ (Additional file 7: Table S1) as described earlier [34]. The fold difference was calculated using the formula $2^{-(\Delta \Delta C T)}$ and normalized with $\beta$-actin levels.

\section{Quantification of RRE-RNA export activity of Rev}

HEK293T cells were transfected with hStau2-59 with lipofectamine LTX plus reagent (Invitrogen, USA) for overexpression. After 24 hours of rest, cells were transfected with equal amount of pNL4-3. After 48 hours of hStau-2 transfection, cells were harvested and cytoplasmic RNA was isolated using Paris kit (Ambion, USA). RNase free DNase I treatment was given to remove DNA contamination. $2 \mu \mathrm{g}$ of RNA from each group were reverse transcribed using SuperScript III Reverse Transcriptase (Invitrogen, USA), random hexamer at $50^{\circ} \mathrm{C}$ for 1 hour followed by inactivation at $70^{\circ} \mathrm{C}$ for $15 \mathrm{~min}$. For absolute real time PCR, standard was made using serial dilution of RRE-GEM construct containing known copy numbers (Additional file 4: Figure S4). The FAM labeled RRE taqman probe was commercially designed and synthesized (Invitrogen, USA). 500 ng of cytoplasmic RNA was reverse transcribed and then PCR amplified using probe, taqman universal mix II with UNG (Invitrogen, USA) and checked for the number of copies of RRE representative of unspliced RNA. UNG glycosylase was added to remove any carryover of PCR. Background reading was checked by analyzing negative RT control. Nuclear contamination in cytoplasmic fraction was checked by PCR amplification by pre-GAPDH primers (Additional file 3: Figure S3).

\section{HIV-1 Rev-hStau-2 protein-protein docking}

The structure of the Rev protein is available in PDB (2X7L), and chain $\mathrm{M}$ of this protein was used to perform the docking study with Staufen homologs. As the structure of hStau-2 is not available, the sequence of hStau-2 was used in a BLASTP search [49] against PDB for finding suitable homologs. The homology search revealed that the N-terminal of the hStau-2 protein (Residues 6 to 70 ) had $39 \%$ sequence similarity with Dsrm domain 
of spermatid perinuclear RNA-binding protein of human (2DMY), amino acid residues $201-275$ had 57\% identity with the Staufen protein from Drosophila (1EKZ) and amino acid residues 308-383 had 96\% identity with the dsRNA binding domain in Staufen homolog (PDB id: 1UHZ) from mouse. Three different docking studies were performed between the known Rev structure and each of the homologs obtained for hStau-2. PatchDock $[50,51]$ server with refinement tool FireDock $[52,53]$ were used for molecular docking. PatchDock provided results which were ranked according to geometric shape complementarity score after molecular shape representation and surface patch matching. These results were used for further refinement and re-scoring of 1000 top scoring complexes using FireDock. The results from Firedock ranked the complexes according to the global energy score. The best docking model with the Staufen homolog of mouse (1UHZ) and HIV-1 Rev (2X7L) is shown in Additional file 5: Figure S5B and Figure 6A. The global energy function of this complex was calculated by FireDock server to be -7.05 of relative units (this value is considered to be related to free binding energy and higher negative value means higher free binding energy and thus higher interaction probability). Using this model, the residues which are forming intermolecular interactions like hydrogen bonds, electrostatic and hydrophobic interactions among these two protein structures were identified. The interacting residues between Rev and hStau-2 for this model were also predicted by cons-PPISP server $[54,55]$. The best ranked docked complexes between Staufen homolog of mouse (1UHZ) and HIV-1 Rev protein (2X7L) suggested that the possible residues of hStau- 2 that can bind HIV-1 Rev resided in the regions $232-235,255-262,314-320$ and $370-375$. Subsequent mutation studies were carried out to validate this prediction. One such hStau-2 mutant, carrying the mutations Q314R, A318F and K319E, was found defective in binding Rev.

\section{Statistical analyses}

All the experiments were performed more than three times or as mentioned in figure legends. Average values of the experiments are plotted and error bars represent \pm SD (standard deviation). Statistical analyses were done with Student's $t$-test and the significance expressed as $\mathrm{p}$ value $\leq 0.05$.

\section{Additional files}

Additional file 1: Figure S1. Identification of human Staufen-2 (hStau-2) as Rev interacting factor through affinity column chromatography. A) Purification of recombinant Histidine tagged Rev protein: Recombinant Rev protein was purified from BL-21 DE3 codon plus (RIL) cells and checked on $15 \%$ SDS PAGE followed by Coomassie Staining. Rev was purified to homogeneity and showed a band $\sim 18 \mathrm{kDa} .1$ and 2 denotes two batches of eluted purified. protein. B) Purified protein was used as bait to pull down Rev interacting factors from SUP-T1 lysates: Representative gel showing fractionation of pull down samples from 3 experiments (T1, T2 and T3). Lanes C1, C2 and C3 represent control experiments where SUP-T1 cell lysates were incubated with Talon resin without Rev. M represent protein marker and rec-Rev represents the purified protein. Arrows indicate the bands excised for MALDI analyses from this gel. The experiment was repeated more than 10 times and protein bands were excised from several gels and given for MALDI analysis. C) Table showing partial list of proteins identified as Rev interacting host factors from affinity chromatography experiments which were repeatedly identified in several pull down assays. The list included Staufen-2.

Additional file 2: Figure S2. Mascot search result and score histogram for Staufen-2.

Additional file 3: Figure S3. Reverse Transcriptase-PCR to check the purity of the cytoplasmic fractions: Cytoplasmic fractions were separated and RNA was isolated using PARIS kit. CDNA was prepared from isolated RNA and amplified by $\beta$-actin and pre-GAPDH primers as mentioned in the Table S1. Absence of pre-GAPDH band in cytoplasmic fraction is indicative of the purity of cytoplasmic fraction which showed amplified $\beta$-actin band and not pre-GAPDH band.

Additional file 4: Figure S4. RRE standard plots. Concentration of RRE template (construct RRE-GEM) was determined spectrophotometrically and the number of copies was determined for each dilution. A) FAM labeled Taqman probe was designed against RRE. Fluorescence intensities of RRE standards were plotted against their respective $C T$ values. B) Standard curve of CT value vs amount. This standard curve was used for determining copy numbers of unknown samples.

Additional file 5: Figure S5. Docking studies to predict hStau-2 amino acid residues that can possibly interact with Rev: $\mathbf{A}$ ) The alignment of hStau- 2 with the mouse homolog of Staufen: The residues which are mutated are highlighted in the alignment. B) The docked complex of Staufen homolog (1UHZ) and Rev protein (2X7L): The HIV-1 Rev protein is depicted in ribbon and hStau-2 protein in spacefill to show the probable binding sites. The interacting residues in Staufen are shown as sticks and are labeled.

Additional file 6: Figure S6. $C T$ value curve for RRE export by wt hStau-2 and hStau-2Mut overexpression: A leftward shift in the CT curve of viral RRE levels upon hStau-2 overexpression (curve A) though no such shift is observed in hStau-2Mut overexpression which remained similar to the control experiment (curve B and C).

Additional file 7: Table S1. Primers used in the study.

\section{Abbreviations}

HIV: Human immunodeficiency virus, (hStau-2): human staufen-2; qRT-PCR: Quantitative Real Time PCR; AIDS: Acquired immune deficiency syndrome; RRE: Rev response element; RNPs: Ribonucleoproteins; EGFP: Enhanced green fluorescent protein; HEK: Human embryonic kidney cells; MALDI-TOF: Matrix-assisted laser desorption/ionization time of flight; ELISA: Enzyme-linked immunosorbent assay; IP: Immunoprecipitation; siRNA: Small interfering RNA; oligos: Oligonucleotides; HRP: Horseradish peroxidase; DAPI: 4',6-diamidino-2-phenylindole; IPTG: Isopropyl $\beta$-D-1thiogalactopyranoside; DTT: Dithiothreitol; UNG: Uracil N-Glycosylase; PDB: Protein Data Bank; PMSF: phenylmethanesulfonylfluoride.

\section{Competing interests}

The authors declare that there is no competing interests.

\section{Authors' contributions}

$A B$ and $S B$ conceived and designed the study; $A B, R B, K B$ and $P G$ performed the experiments; $A B, R B$ and $S B$ analyzed the data and wrote the paper. All authors have read and approved the final manuscript.

\section{Acknowledgements}

We thank Nalini M (Confocal Imaging) and Monica K (Proteomics), UoH. We thank Dr. Mitra, DRILS for siRNA designing, Prof. Kondapi, UoH for virus culture facility and Dr Mande, NCCS, Pune for intellectual inputs. DBT-CREB and DST-PURSE grants to the Department of Biochemistry and School of Life Sciences, University of Hyderabad is acknowledged. 


\section{Funding}

This study was supported by grants from DBT, India (BT/PR10092/BRB/10/ $585 / 2007$ and BT/PR3260/BRB/10/967/2011) to SB. AB, RB and KB are funded by research fellowships from Council of Scientific and Industrial Research, University Grants Commission and Department of Biotechnology (DBT-IYBA grant to SB) respectively.

\section{Author details}

'Department of Biochemistry, School of Life Sciences, University of Hyderabad, Gachibowli, Hyderabad, Andhra Pradesh 500046, India. ${ }^{2}$ Department of Biotechnology, University of Pune, Ganeshkhind, Pune, Maharashtra 411007, India.

Received: 9 December 2013 Accepted: 5 February 2014

Published: 13 February 2014

\section{References}

1. Benjamin R, Banerjee A, Sunder SR, Gaddam S, Valluri VL, Banerjee S: Discordance in CD4 + T-cell levels and viral loads with Co-occurrence of elevated peripheral TNF-alpha and IL-4 in newly diagnosed HIV-TB Co-infected cases. PLoS One 2013, 8:e70250.

2. Wahl SM, Greenwell-Wild T, Peng G, Hale-Donze H, Orenstein JM: Co-infection with opportunistic pathogens promotes human immunodeficiency virus type 1 infection in macrophages. J Infect Dis 1999, 179(Suppl 3):S457-S460.

3. Gill PS: Pathogenesis of HIV-related malignancies. Curr Opin Oncol 1991, 3:867-871

4. French M, Keane N, McKinnon E, Phung S, Price P: Susceptibility to opportunistic infections in HIV-infected patients with increased CD4 T-cell counts on antiretroviral therapy may be predicted by markers of dysfunctional effector memory CD4 T cells and B cells. HIV Med 2007, 8:148-155.

5. Pollard WW, Malim MH: The HIV-1 Rev protein. Annu Rev Microbiol 1998, 52:491-532.

6. Zenklusen D, Stutz F: Nuclear export of mRNA. FEBS Lett 2001, 498:150-156.

7. Karn J, Stoltzfus CM: Transcriptional and posttranscriptional regulation of HIV-1 gene expression. Cold Spring Harb Perspect Med 2012, 2:a006916.

8. Malim MH, Hauber J, Le SY, Maizel JV, Cullen BR: The HIV-1 rev trans-activator acts through a structured target sequence to activate nuclear export of unspliced viral mRNA. Nature 1989, 338:254-257.

9. Bogerd HP, Fridell RA, Madore S, Cullen BR: Identification of a novel cellular cofactor for the Rev/Rex class of retroviral regulatory proteins. Cell 1995, 82:485-494.

10. Kula A, Guerra J, Knezevich A, Kleva D, Myers MP, Marcello A: Characterization of the HIV-1 RNA associated proteome identifies Matrin 3 as a nuclear cofactor of Rev function. Retrovirology 2011, 8:60

11. Fridell RA, Bogerd HP, Cullen BR: Nuclear export of late HIV-1 mRNAs occurs via a cellular protein export pathway. Proc Natl Acad Sci USA 1996, 93:4421-4424.

12. Kula A, Gharu L, Marcello A: HIV-1 pre-mRNA commitment to Rev mediated export through PSF and Matrin 3. Virology 2013, 435:329-340.

13. Gharu L, Kula A, Bardina M, Marcello A: Post transcriptional regulation of HIV-1 gene expression by MATR3, PSF and Rev. Retrovirology 2013, 10:S21-S31.

14. Yedavalli VSRK, Jeang KT: Matrin 3 is a co-factor for HIV-1 Rev in regulating post-transcriptional viral gene expression. Retrovirology 2011, 8:61.

15. Naji S, Ambrus G, Cimermancic P, Reyes JR, Johnson JR, Filbrandt R, Huber MD, Vesely P, Krogan NJ, Yates JR III, et al: Host cell interactome of HIV-1 Rev includes RNA helicases involved in multiple facets of virus production. Mol Cell Proteomics 2012, 11:M111. 015313.

16. Bogerd HP, Echarri A, Ross TM, Cullen BR: Inhibition of human immunodeficiency virus Rev and human T-cell leukemia virus Rex function, but not Mason-Pfizer monkey virus constitutive transport element activity, by a mutant human nucleoporin targeted to Crm1. J Virol 1998, 72:8627-8635.

17. Ariumi $Y$, Trono D: Ataxia-telangiectasia-mutated (ATM) protein can enhance human immunodeficiency virus type 1 replication by stimulating Rev function. J Virol 2006, 80:2445-2452.

18. Bevec $D$, Jaksche H, Oft M, Wohl T, Himmelspach M, Pacher A, Schebesta M, Koettnitz K, Dobrovnik M, Csonga R, et al: Inhibition of HIV-1 replication in lymphocytes by mutants of the Rev cofactor elF-5A. Science 1996, 271:1858-1860.
19. Reddy TR, Xu W, Mau JK, Goodwin CD, Suhasini M, Tang H, Frimpong K, Rose DW, Wong-Staal F: Inhibition of HIV replication by dominant negative mutants of Sam68, a functional homolog of HIV-1 Rev. Nat Med 1999, 5:635-642

20. Fang J, Kubota S, Yang B, Zhou N, Zhang H, Godbout R, Pomerantz RJ: A DEAD box protein facilitates HIV-1 replication as a cellular co-factor of Rev. Virology 2004, 330:471-480.

21. Modem S, Reddy TR: An anti-apoptotic protein, Hax-1, inhibits the HIV-1 rev function by altering its sub-cellular localization. J Cell Physiol 2008, 214:14-19.

22. Xu Y, Reddy TR, Fischer WH, Wong-Staal F: A Novel hnRNP Specifically Interacts with HIV-1 RRE RNA. J Biomed Sci 1996, 3:82-91.

23. Powell DM, Amaral MC, Wu JY, Maniatis T, Greene WC: HIV Rev-dependent binding of SF2/ASF to the Rev response element: possible role in Rev-mediated inhibition of HIV RNA splicing. Proc Natl Acad Sci USA 1997, 94:973-978.

24. Banerjee A, Benjamin R, Banerjee S: Impact of viral factors on subcellular distribution and RNA export activity of HIV-1 Rev in astrocytes $1321 \mathrm{~N} 1$. PLoS One 2013, 8:e72905.

25. Jager S, Cimermancic P, Gulbahce N, Johnson JR, McGovern KE, Clarke SC, Shales M, Mercenne G, Pache L, Li K, et al: Global landscape of HIV-human protein complexes. Nature 2012, 481:365-370.

26. Macchi P, Brownawell AM, Grunewald B, DesGroseillers L, Macara IG, Kiebler MA: The brain-specific double-stranded RNA-binding protein Staufen2: nucleolar accumulation and isoform-specific exportin-5-dependent export. J Biol Chem 2004, 279:31440-31444.

27. Wickham L, Duchaine T, Luo M, Nabi IR, DesGroseillers L: Mammalian staufen is a double-stranded-RNA- and tubulin-binding protein which localizes to the rough endoplasmic reticulum. Mol Cell Biol 1999, 19:2220-2230.

28. Duchaine TF, Hemraj I, Furic L, Deitinghoff A, Kiebler MA, DesGroseillers L: Staufen2 isoforms localize to the somatodendritic domain of neurons and interact with different organelles. J Cell Sci 2002, 115:3285-3295.

29. Miki T, Kamikawa Y, Kurono S, Kaneko Y, Katahira J, Yoneda Y: Cell typedependent gene regulation by Staufen2 in conjunction with Upf1. BMC Mol Biol 2011, 12:48.

30. Thomas MG, Martinez Tosar LJ, Loschi M, Pasquini JM, Correale J, Kindler S, Boccaccio GL: Staufen recruitment into stress granules does not affect early mRNA transport in oligodendrocytes. Mol Biol Cell 2005, 16:405-420

31. Hope TJ: The ins and outs of HIV Rev. Arch Biochem Biophys 1999, 365:186-191.

32. Miki T, Yoneda Y: Alternative splicing of Staufen2 creates the nuclear export signal for CRM1 (Exportin 1). J Biol Chem 2004, 279:47473-47479.

33. Chatel-Chaix L, Abrahamyan L, Frechina C, Mouland AJ, DesGroseillers L: The host protein Staufen1 participates in human immunodeficiency virus type 1 assembly in live cells by influencing pr55Gag multimerization. J Virol 2007, 81:6216-6230.

34. Chatel-Chaix L, Clement JF, Martel C, Beriault V, Gatignol A, DesGroseillers L, Mouland AJ: Identification of Staufen in the human immunodeficiency virus type $1 \mathrm{Gag}$ ribonucleoprotein complex and a role in generating infectious viral particles. Mol Cell Biol 2004, 24:2637-2648.

35. Abrahamyan LG, Chatel-Chaix L, Ajamian L, Milev MP, Monette A, Clement JF, Song R, Lehmann M, DesGroseillers L, Laughrea M, et al: Novel Staufen 1 ribonucleoproteins prevent formation of stress granules but favour encapsidation of HIV-1 genomic RNA. J Cell Sci 2010, 123:369-383.

36. Thomas MG, Martinez Tosar L, Desbats MA, Leishman CC, Boccaccio GL: Mammalian Staufen 1 is recruited to stress granules and impairs their assembly. J Cell Sci 2009, 122:563-573.

37. Falcon AM, Fortes $\mathrm{P}$, Marion RM, Beloso A, Ortin J: Interaction of influenza virus NS1 protein and the human homologue of Staufen in vivo and in vitro. Nucleic Acids Res 1999, 27:2241-2247.

38. Furic L, Maher-Laporte M, DesGroseillers L: A genome-wide approach identifies distinct but overlapping subsets of cellular mRNAs associated with Staufen1-and Staufen2-containing ribonucleoprotein complexes. RNA 2008, 14:324-335.

39. Tosar LJ, Thomas MG, Baez MV, Ibanez I, Chernomoretz A, Boccaccio GL: Staufen: from embryo polarity to cellular stress and neurodegeneration. Front Biosci (Schol Ed) 2012, 4:432-452.

40. Fornerod M, Ohno M, Yoshida M, Mattaj IW: CRM1 is an export receptor for leucine-rich nuclear export signals. Cell 1997, 90:1051-1060. 
41. Sanchez-Velar N, Udofia EB, Yu Z, Zapp ML: hRIP, a cellular cofactor for Rev function, promotes release of HIV RNAs from the perinuclear region. Genes Dev 2004, 18:23-34.

42. Yu Z, Sanchez-Velar N, Catrina IE, Kittler EL, Udofia EB, Zapp ML: The cellular HIV-1 Rev cofactor hRIP is required for viral replication. Proc Natl Acad Sci USA 2005, 102:4027-4032.

43. Chiu YL, Greene WC: APOBEC3G: an intracellular centurion. Philos Trans $R$ Soc Lond B Biol Sci 2009, 364:689-703.

44. Chiu YL, Witkowska HE, Hall SC, Santiago M, Soros VB, Esnault C, Heidmann T, Greene WC: High-molecular-mass APOBEC3G complexes restrict Alu retrotransposition. Proc Natl Acad Sci USA 2006, 103:15588-15593.

45. Kutner RH, Zhang XY, Reiser J: Production, concentration and titration of pseudotyped HIV-1-based lentiviral vectors. Nat Protoc 2009, 4:495-505.

46. Wingfield PT, Stahl SJ, Payton MA, Venkatesan S, Misra M, Steven AC: HIV-1 Rev expressed in recombinant Escherichia coli: purification, polymerization, and conformational properties. Biochemistry 1991, 30:7527-7534

47. Fankhauser C, Izaurralde E, Adachi Y, Wingfield P, Laemmli UK: Specific complex of human immunodeficiency virus type 1 rev and nucleolar B23 proteins: dissociation by the Rev response element. Mol Cell Biol 1991, 11:2567-2575

48. Chevallet $M$, Luche $S$, Rabilloud $T$ : Silver staining of proteins in polyacrylamide gels. Nat Protoc 2006, 1:1852-1858.

49. Altschul SF, Madden TL, Schaffer AA, Zhang J, Zhang Z, Miller W, Lipman DJ: Gapped BLAST and PSI-BLAST: a new generation of protein database search programs. Nucleic Acids Res 1997, 25:3389-3402.

50. Duhovny D, Nussinov R, Wolfson HJ: Efficient unbound docking of rigid molecules. Lect Notes Comput Sc 2002, 2452:185-200.

51. Schneidman-Duhovny D, Inbar Y, Nussinov R, Wolfson HJ: PatchDock and SymmDock: servers for rigid and symmetric docking. Nucleic Acids Res 2005, 33:W363-W367.

52. Andrusier $\mathrm{N}$, Nussinov $\mathrm{R}$, Wolfson $\mathrm{HJ}$ : FireDock: fast interaction refinement in molecular docking. Proteins 2007, 69:139-159.

53. Mashiach E, Schneidman-Duhovny D, Andrusier N, Nussinov R, Wolfson HJ: FireDock: a web server for fast interaction refinement in molecular docking. Nucleic Acids Res 2008, 36:W229-W232.

54. Chen $\mathrm{H}$, Zhou HX: Prediction of interface residues in protein-protein complexes by a consensus neural network method: test against NMR data. Proteins 2005, 61:21-35.

55. Zhou HX, Shan Y: Prediction of protein interaction sites from sequence profile and residue neighbor list. Proteins 2001, 44:336-343.

doi:10.1186/1742-4690-11-18

Cite this article as: Banerjee et al:: Human protein Staufen-2 promotes HIV-1 proliferation by positively regulating RNA export activity of viral protein Rev. Retrovirology 2014 11:18.

\section{Submit your next manuscript to BioMed Central and take full advantage of:}

- Convenient online submission

- Thorough peer review

- No space constraints or color figure charges

- Immediate publication on acceptance

- Inclusion in PubMed, CAS, Scopus and Google Scholar

- Research which is freely available for redistribution 